\title{
Integration of Porous Carbon Nanowrinkles into Carbon Micropost Array for Microsupercapacitors
}

\author{
Shuang Xi $(\mathbb{D}$, Ying Liu $(\mathbb{D}$, Yinlong Zhu, and Yutu Yang (i) \\ School of Mechanical and Electronic Engineering, Nanjing Forestry University, Nanjing 210037, China \\ Correspondence should be addressed to Shuang Xi; shuangxi@hust.edu.cn
}

Received 25 October 2017; Accepted 26 December 2017; Published 22 January 2018

Academic Editor: Hassan Karimi-Maleh

Copyright (c) 2018 Shuang Xi et al. This is an open access article distributed under the Creative Commons Attribution License, which permits unrestricted use, distribution, and reproduction in any medium, provided the original work is properly cited.

\begin{abstract}
Porous carbon nanowrinkles (PCW) coated on carbon micropost (CMP) arrays were successfully fabricated via three-step process, which took advantages of the large difference in elastic moduli between PCW and the raw material of CMP. The effect of nanowrinkle integration on the electrochemical performances was investigated, showing an improved electrochemical performance. The electrode also shows excellent cycling stability, which retains $84 \%$ of its initial discharge capacitance after 1700 cycles with $>90 \%$ Coulombic efficiency. This enhanced electrochemical performance is ascribed to the synergistic effect of enlarged surface area and porous structure of PCW. The obtained PCW/CMP compositing electrode with the advantages of low cost and easy scaling-up has great potential for on-chip supercapacitors.
\end{abstract}

\section{Introduction}

With the fast development of miniaturized electronic systems, miniaturized energy-storage/retrieval devices based on batteries and electrochemical capacitors have attracted increasing attention [1-3]. Among the energy-storage devices, miniaturized supercapacitors (microsupercapacitors) have gained ample interest because they possess high power density, rapid charge/discharge rates, and superior cycling stability/durability, which makes them probably the most important next-generation energy storage devices $[4,5]$. According to the charge storage mechanism, supercapacitors can be classified into two broad categories, that is, electrical double layered capacitor (EDLC) and pseudocapacitor. Contrary to the charge storage process in pseudocapacitance, which is based on a fast faradic reaction at the near surface of electrodes, the capacitance of EDLCs arises from electrostatic separation at the interface of carbon-based electrodes based on the non-Faradaic reaction [6, 7]. Generally, EDLCs have higher rate capability, higher power density, and prolonged cyclic life compared with pseudocapacitors [8]. For EDLCs, large specific surface area of electrode materials, such as carbon nanotubes [9], onion-like carbon [10], activated carbon [11], or graphene [12], plays an importance role.
It has been reported that capacitors constructed with porous carbons demonstrate high specific capacitance as well as high power density, wherein the charge is stored at the electrode/electrolyte interface through reversible ion adsorption at high-surface-area porous carbon electrodes [13]. For this kind of electrode, one of the main technological issues in the fabrication process is ensuring the strong adhesion between nanoporous carbon and the current collector [14].

Previously, our group has provided a facile approach to fabricate porous carbon nanowrinkles (PCW) on threedimensional carbon microposts (CMP) by photolithography SU-8 photoresist, sputtering carbon film, and the following pyrolysis [15]. During pyrolysis, severe shrinkage occurred when SU-8 photoresist gradually converted into glassy CMP, and at the same time wrinkle patterns were generated in the thin hard carbon film supported on compliant SU-8 substrate due to their big difference in elastic modulus. The obtained structure proved to be stably integrated considering the same intrinsic nature of wrinkles and microposts. In this work, the PCW/CMP compositing structure was used as the electrode of microsupercapacitors. The resulting hierarchical electrode combining good electrical conductivity of CMP and porous nature of PCW, also with strong adhesion between hybrid 
PCW and CMP arrays avoiding the utilize of binders, yields promising capacitive performance due to fast penetration of the electrolyte.

\section{Experiment Details}

2.1. Integration of $P C W$ into $C M P$. The procedures for the preparation of the PCW/CMP composite have been reported previously [15]: briefly, photolithography of SU-8 (GM 1075) photoresist to obtain polymer micropost array, followed by a thin carbon layer depositing on it through ion sputtering using ion sputter coater, and then pyrolyzing the sample in an alumina tube furnace $\left(300^{\circ} \mathrm{C}\right.$ for $30 \mathrm{~min}$, then $1000^{\circ} \mathrm{C}$ for $1 \mathrm{~h}$ ) to yield PCW/CMP integrating structures. During pyrolysis process, the SU-8 pattern was decomposed into carbon arrays, degassing of large quantities of vapor (such as $\mathrm{CO}, \mathrm{CH}_{4}$, and $\mathrm{H}_{2} \mathrm{O}$ ), leading to a strong shrinkage of the pristine pattern geometry. Due to the big difference in the Young's modulus value of SU-8 and carbon material, wrinkle patterns occur in the sputtered carbon thin layer.

2.2. Characterization. The morphologies of the samples were characterized by field emission scanning electron microscopy (FESEM, Hitachi, S-4800, Japan). The structures properties of wrinkles were examined by transmission electron microscope (TEM, FEI-F20) equipped with energy-dispersive Xray (EDX) spectrometry and selected-area electron diffraction (SAED). Cyclic voltammetry (CV) properties were examined by Autolab work station (PGSTAT-302 N, Eco Echemie B.V. Company, Utrecht, Netherlands) in $1 \mathrm{M} \mathrm{Na}_{2} \mathrm{SO}_{4}$ aqueous solution. Galvanostatic charging/discharging and cycling tests were conducted using a battery measurement system (LAND CT2001A, Wuhan LAND Electronics, Wuhan, China). All the electrochemical experiments were conducted at room temperature. To conduct these electrochemical experiments, a current collector layer was prepared in advance, which was realized by spinning coating a positive photoresist (AZ5214) on a silicon substrate at $500 \mathrm{rpm}$ for $5 \mathrm{~s}$ and then at $4000 \mathrm{rpm}$ for $30 \mathrm{~s}$, followed by a $3 \mathrm{~min}$ soft bake at $95^{\circ} \mathrm{C}$. A glassy carbon layer collector of $\sim 1 \mu \mathrm{m}$ thickness was obtained by a pyrolysis of the photoresist film at $1000^{\circ} \mathrm{C}$ (heating rate $10^{\circ} \mathrm{C} / \mathrm{min}$ ) for $60 \mathrm{~min}$ in forming gas $\left(5 \% \mathrm{H}_{2}\right.$ in $\mathrm{N}_{2}$ ) atmosphere.

\section{Results and Discussion}

3.1. Morphologies and Structures. Upon depositing the carbon film on SU-8 post arrays, a wrinkle pattern forms in order to release the strain energy of the carbon film accumulated by residual compression, as shown in Figure 1. From Figures 1(a) and 1(b), which show the top view of carbon wrinkles on cylindrical posts, it can be observed that the pyrolyzed cylindrical pillar is about $20 \mu \mathrm{m}$ in diameter; the grooves and ridges of the wrinkles are about tens of nanometer in width. Since the top surface of the carbon post is cylindrically symmetrical at the circle center, patterns near the center look like labyrinthine due to isotropic shrinkage, while patterns near the edge appear to be spoke-like due to the free boundary
[16]. Wrinkle patterns on the lateral surface of carbon posts are shown in Figures $1(\mathrm{c})$ and $1(\mathrm{~d})$, demonstrating that the width of the wrinkle patterns is similar to that on the top surface. What is different, patterns in the lateral surface are approximately distributed along the vertical direction, which might have been resulted from the shrinking orientation of polymer posts.

To pursue detailed structure of the wrinkles on carbon posts, TEM characterization was conducted. The mesoporous feature of the wrinkles is clearly revealed through TEM analysis, as shown in Figure 2. The low-magnification TEM image shown in Figure 2(a) demonstrates several pieces of wrinkle sheets peeled off from the carbon post via high power ultrasonication, which exhibit folding silk-like morphology with transparent feature, indicating their ultrathin nature. The grooves and ridges of wrinkles are clearly observed, and the lateral size of the wrinkle sheets is much larger than their thickness, revealing a typical two-dimensional nanostructure. EDX analyses shown as an inset demonstrate their chemical composition mainly consisting of C elements, while few $\mathrm{O}$ elements could come from impurities in pyrolyzed carbon post, and the weak Ni peak arose from the nickel mesh on which the sample is loaded. The enlarged image shown in Figure 2(b) reveals that the nanosheets are around several nanometers in thickness and also confirms their porous, ultrathin, and wrinkle feature. This characterization is ideal for intercalation-based electrode materials because of the high surface area as well as the short ion diffusion path. The corresponding SAED pattern shown as an inset of Figure 2(b) indicates the amorphous structure of carbon nanowrinkles. The porous feature of carbon wrinkle nanosheets will provide good access for electrolyte ions across the interface, thus accelerating ion transport. Moreover, the wrinkle texture could help to release the mechanical strain caused by volume expending/shrinking during the repeated ion insertion-extraction, which will be beneficial to improve the electrochemical performance.

3.2. Electrochemical Properties. For the prepared PCW/CMP structure, it is difficult to peel off the wrinkle nanosheets from carbon posts, indicating the strong adhering force between these two composites. Considering the good connection between the porous wrinkles and substrate, the obtained PCW/CMP hybrid structure can be directly used as a binder-free electrode for supercapacitor to estimate its electrochemical properties. The specific CV curves of the carbon post and PCW/CMP electrodes with size of $1 \mathrm{~cm}^{2}$ are shown in Figure 3(a) at the scan rate of $50 \mathrm{mV} / \mathrm{s}$ in $1 \mathrm{M} \mathrm{Na}_{2} \mathrm{SO}_{4}$ electrolyte. The $\mathrm{CV}$ integrated area of pristine CMP array is apparently smaller than that of PCW/CMP hybrid electrode, indicating reduced capacitance. The specific geometric capacitance $\left(C_{A}\right)$ values can be calculated based on the $\mathrm{CV}$ results, according to the following equation [17]:

$$
C_{A}=\frac{\int i(v) d v}{2 S V},
$$

where $S$ is the potential sweep rate $(\mathrm{V} / \mathrm{s})$ and $V$ represents the potential range. The specific geometric capacitance of 


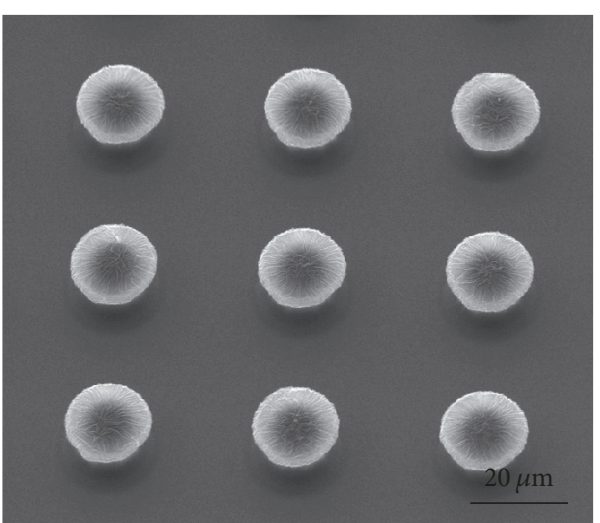

(a)

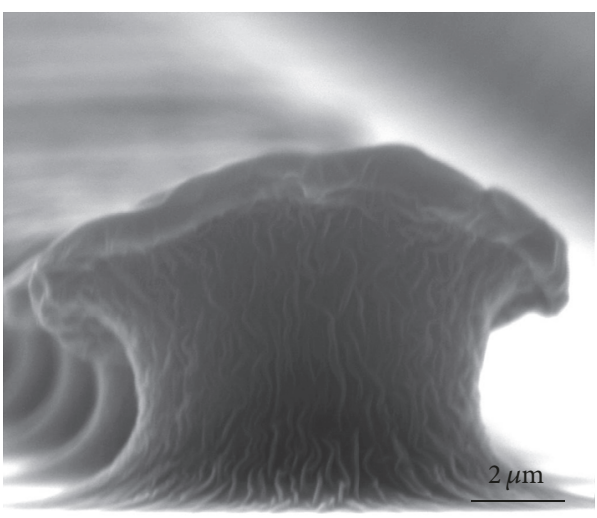

(c)

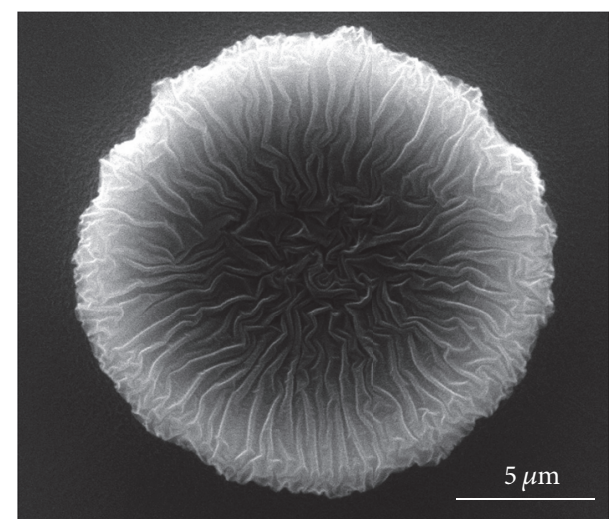

(b)

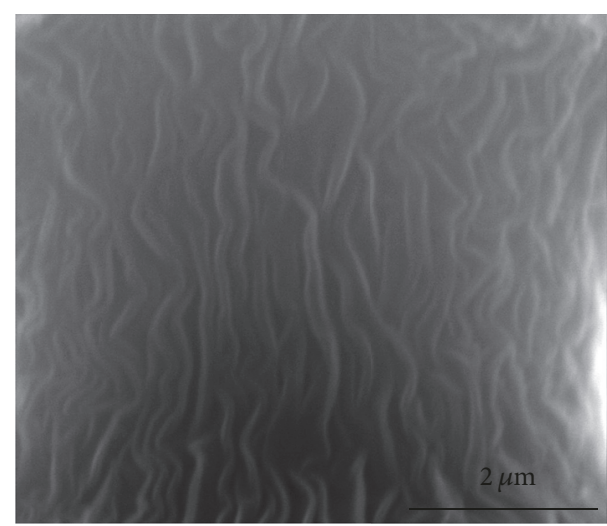

(d)

FIGURE 1: SEM images of wrinkle patterns of carbon film on CMP. (a) Top view of arrays; (b) top view of single post; (c) side view of arrays; (d) side view of single post.

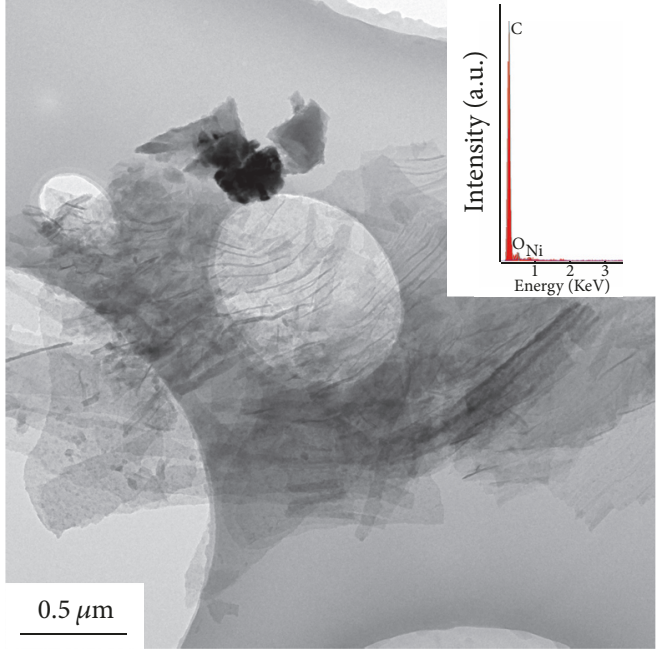

(a)

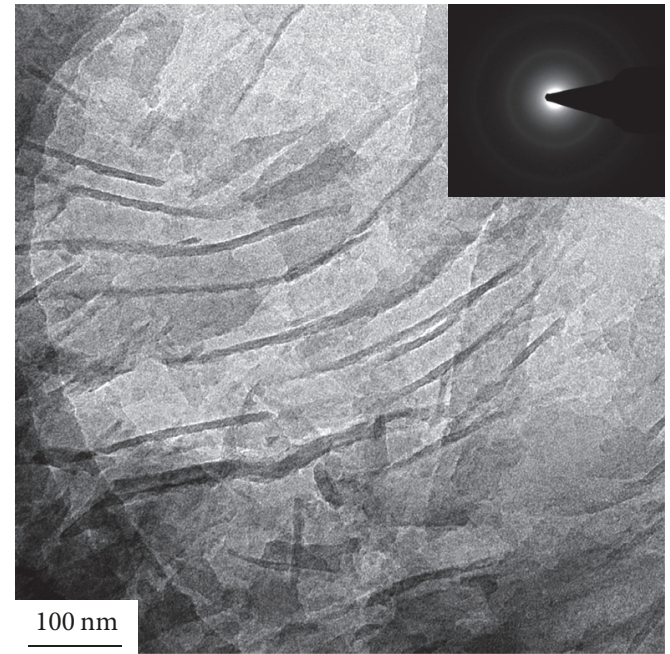

(b)

FIGURE 2: TEM images of wrinkle structures peeled off from the carbon posts. (a) Low-magnification TEM image, with the EDX spectrum as an inset; (b) magnified TEM image, with the corresponding SEAD pattern as an inset. 


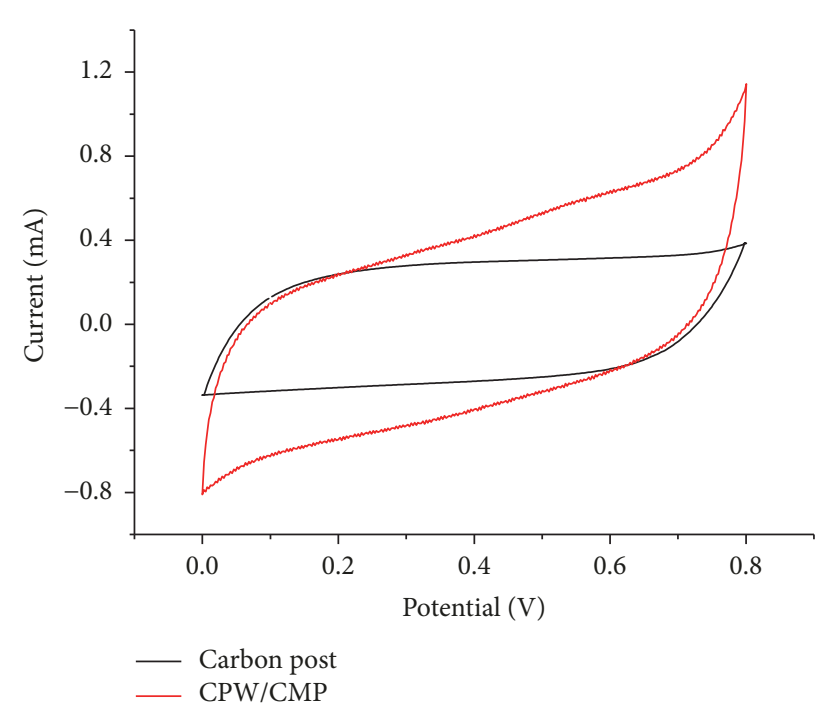

(a)

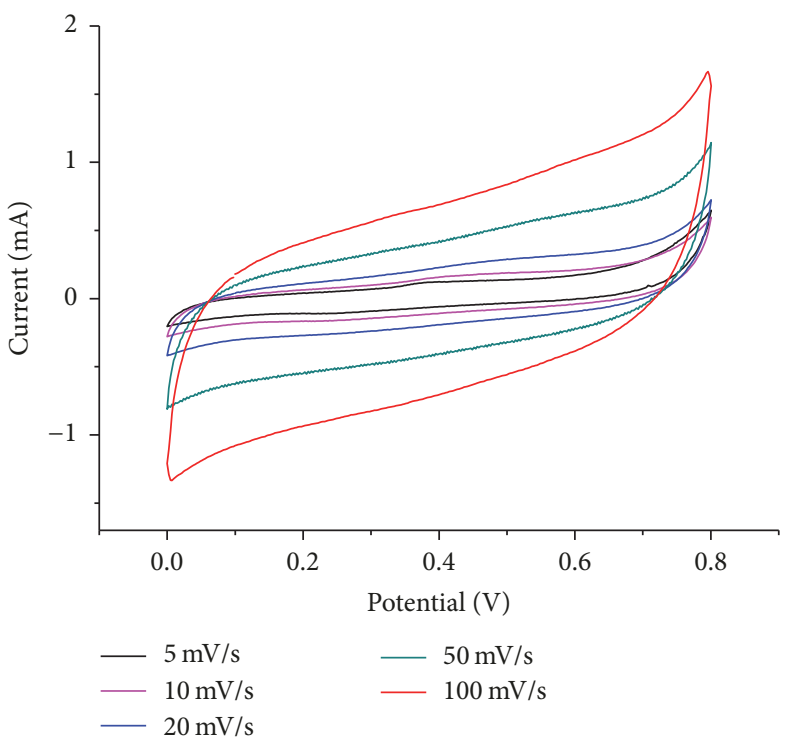

(b)

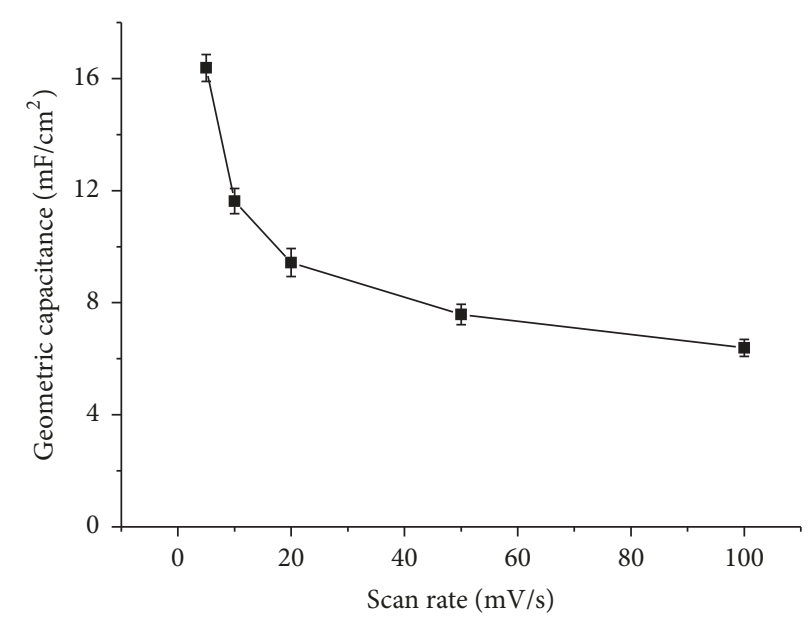

(c)

Figure 3: (a) CV of the pure carbon post arrays and PCW/CMP composites at a scan rate of $50 \mathrm{mV} / \mathrm{s}$ in $1 \mathrm{M} \mathrm{Na}_{2} \mathrm{SO}_{4}$ electrolyte. (b) CV of the PCW/CMP at various scan rates from $5 \mathrm{mV} / \mathrm{s}$ to $100 \mathrm{mV} / \mathrm{s}$. (c) Geometric capacitance as a function of scan rate.

$\mathrm{PCW} / \mathrm{CMP}$ is $7.58 \mathrm{mF} / \mathrm{cm}^{2}$ at the scan rate of $50 \mathrm{mV} / \mathrm{s}$, which is nearly two times higher than that of carbon posts due to increased surface area and typical porous structure. The main reason of the enhanced specific capacitance is that the introduction of PCW creates a large and electrochemically accessible internal surface area, which thus increases the double layer capacitance. Figure 3(b) shows the CV curves of a PCW/CMP sample at various scan rates. The CV curves possess a near symmetric rectangular shape and exhibit near mirror-image current response on voltage reversal, revealing a fast and reversible reaction along with the excellent capacitive performance of PCW/CMP electrode. Figure 3(c) shows the specific geometric capacitance of PCW/CMP electrodes with experimental error bar measured at different scan rates of $5,10,20,50$, and $100 \mathrm{mV} / \mathrm{s}$. The specific capacitance decreases gradually with the scan rate increasing, and the highest value of geometric capacitance is $~ 16.38 \mathrm{mF} / \mathrm{cm}^{2}$ at the lowest scan rate of $5 \mathrm{mV} / \mathrm{s}$.
The electrochemical performance of PCW/CMP electrode was further investigated by galvanostatic charging/discharging technique, with the charging/discharging curves at various current densities in a voltage range of $0-0.7 \mathrm{~V}$ as shown in Figure 4(a). It could be observed that the discharge curves are approximately symmetric with their corresponding charge counterparts, indicating that the composite electrode has typical capacitive properties [18]. The specific capacitance obtained from the discharging curves is calculated according to the following equation [19]:

$$
C=\frac{I \times \Delta t}{\Delta V}
$$

where $I\left(\mathrm{~mA} / \mathrm{cm}^{2}\right)$ is the applied current density, $\Delta t(\mathrm{~s})$ is the discharge time, and $\Delta V(\mathrm{~V})$ is the sweep potential range.

Figure 4(b) shows the variation of the specific geometric capacitance of PCW/CMP electrode at various discharge rates. The geometric capacitance is $\sim 12.8 \mathrm{mF} / \mathrm{cm}^{2}$ 


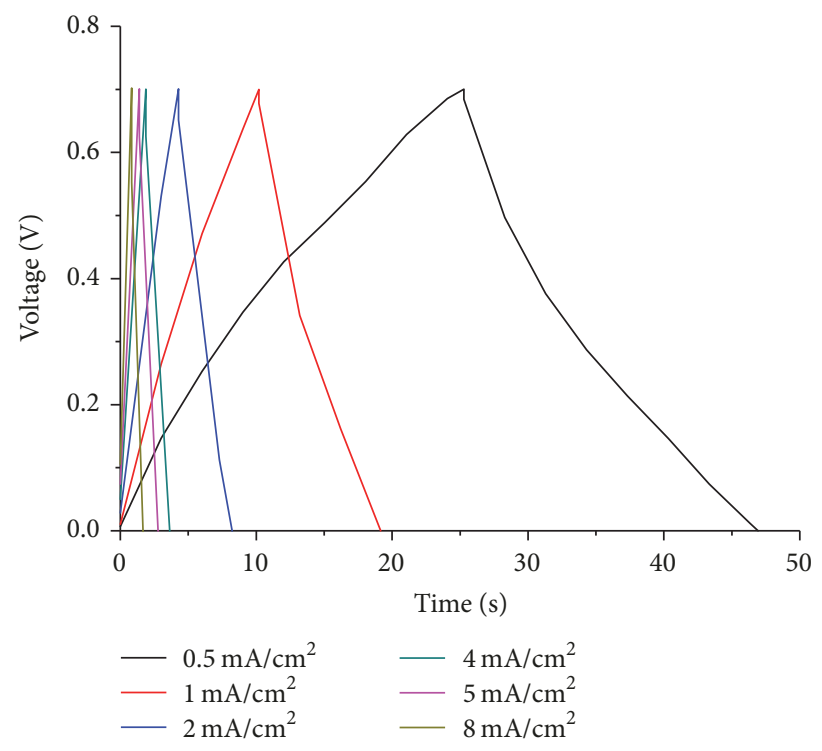

(a)

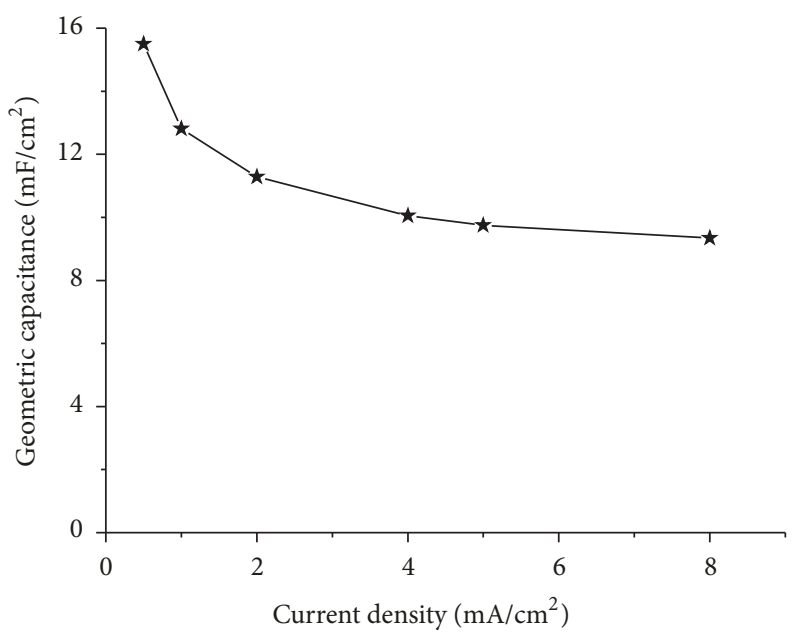

(b)

FIGURE 4: (a) Galvanostatic charging/discharging curves of PCW/CMP electrode at different current density. (b) The specific geometric capacitance of PCW/CMP electrode at various discharge current densities.

at the current density of $1 \mathrm{~mA} / \mathrm{cm}^{2}$. With the charging current density increasing, the specific capacitance of the sample decreases distinctly from $15.5 \mathrm{mF} / \mathrm{cm}^{2}$ (at current density of $0.5 \mathrm{~mA} / \mathrm{cm}^{2}$ ) to $9.34 \mathrm{mF} / \mathrm{cm}^{2}$ (at current density of $8 \mathrm{~mA} / \mathrm{cm}^{2}$ ). The decline of specific capacitance could have been resulted from the shorter diffusion time for protons or ions insertion/extraction at a higher scan rate. These results are in good agreement with those values calculated through CV method. Compared with previously reported works on carbon-based electrochemical microcapacitors, the capacitive performance in this study may be not more advantageous than them, whereas our hybrid electrode has superior rate capability with the electrode maintaining $60 \%$ retention of its initial specific capacitance measured at a high rate of $8 \mathrm{~mA} / \mathrm{cm}^{2}$. This value is superior to many carbonbased electrodes, such as the electrochemically activated carbon microelectrode arrays (capacitance retention of $46 \%$ from $0.5 \mathrm{~mA} / \mathrm{cm}^{2}$ to $5 \mathrm{~mA} / \mathrm{cm}^{2}$ [1]), self-adhesive carbon grains added with $6 \mathrm{wt} \%$ of $\mathrm{KOH}$-treated multilayer graphene (18\% retention from $1 \mathrm{mV} / \mathrm{s}$ to $100 \mathrm{mV} / \mathrm{s}$ [20]).

Besides high specific capacitance, good cycling performance is also an important property for microsupercapacitors. The long-term stability of the PCW/CMP electrode was tested by galvanostatic charge/discharge cycling at a current density of $1 \mathrm{~mA} / \mathrm{cm}^{2}$ for 1700 cycles. Figure 5 displays the specific capacitance retention and Coulombic efficiency of the hybrid electrode as a function of charge/discharge cycling number. From this figure, the Coulombic efficiency keeps $>90 \%$ in the whole testing range. The capacitance retention decreases by $\sim 16 \%$ of the initial value over the first 400 cycles and then remains quite stable for the last 1300 cycles. This indicates acceptable cyclability of PCW/CMP

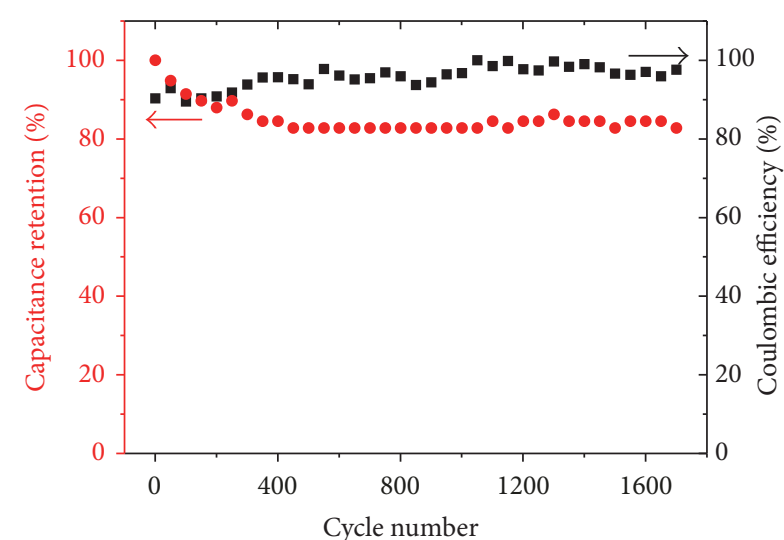

FIGURE 5: Cycling performance and Coulombic efficiency of the electrode up to 1700 cycles at the current density of $1 \mathrm{~mA} / \mathrm{cm}^{2}$.

microelectrodes compared to electrochemical microcapacitors reported by Cui et al., where $62.9 \%$ of the initial capacitance is retained after 2000 cycles [21]. The excellent cycling performance for PCW/CMP ensures the good structural stability as well as firm contact between porous wrinkles and carbon microposts, which would be advantageous for the application in microsupercapacitors.

\section{Conclusion}

In summary, porous carbon nanowrinkles were successfully integrated into carbon micropost substrates via threestep process for on-chip electrochemical microcapacitors. Electrochemical characterization of the integrating structure 
demonstrates improved specific capacitance compared with pure carbon micropost, as well as good rate capability and superior cyclic stability. These excellent electrochemical properties could be attributed to the increased and ion-accessible surface area created by the porous carbon nanowrinkles. The prepared electrode may have promising potential as the high-performance microsupercapacitors.

\section{Conflicts of Interest}

The authors declare that they have no conflicts of interest.

\section{Acknowledgments}

This work is financially supported by Natural Science Foundation of Jiangsu Province (BK20160934) and Youth Science \& Technology Innovation Fund of Nanjing Forestry University (CX2017008).

\section{References}

[1] M. Beidaghi, W. Chen, and C. Wang, "Electrochemically activated carbon micro-electrode arrays for electrochemical microcapacitors," Journal of Power Sources, vol. 196, no. 4, pp. 24032409, 2011.

[2] J. Ren, L. Li, C. Chen et al., "Twisting carbon nanotube fibers for both wire-shaped micro-supercapacitor and micro-battery," Advanced Materials, vol. 25, no. 8, pp. 1155-1159, 2013.

[3] D. Aradilla, F. Gao, G. Lewes-Malandrakis et al., "Powering electrodes for high performance aqueous micro-supercapacitors: diamond-coated silicon nanowires operating at a wide cell voltage of 3V," Electrochimica Acta, vol. 242, pp. 173-179, 2017.

[4] Z. Li, M. Shao, L. Zhou et al., "A flexible all-solid-state microsupercapacitor based on hierarchical CuO@ layered double hydroxide core-shell nanoarrays," Nano Energy, vol. 20, pp. 294304, 2016.

[5] P. Huang, C. Lethien, S. Pinaud et al., "On-chip and freestanding elastic carbon films for micro-supercapacitors," Science, vol. 351, no. 6274, pp. 691-695, 2016.

[6] T. Brousse, D. Bélanger, and J. W. Long, "To be or not to be pseudocapacitive?" Journal of The Electrochemical Society, vol. 162, no. 5, pp. A5185-A5189, 2015.

[7] P. J. Hall, M. Mirzaeian, S. I. Fletcher et al., "Energy storage in electrochemical capacitors: designing functional materials to improve performance," Energy \& Environmental Science, vol. 3, no. 9, pp. 1238-1251, 2010.

[8] P. Simon and Y. Gogotsi, "Materials for electrochemical capacitors," Nature Materials, vol. 7, no. 11, pp. 845-854, 2008.

[9] B. Hsia, J. Marschewski, S. Wang et al., "Highly flexible, all solidstate micro-supercapacitors from vertically aligned carbon nanotubes," Nanotechnology, vol. 25, no. 5, Article ID 055401, 2014.

[10] D. Pech, M. Brunet, H. Durou et al., "Ultrahigh-power micrometre-sized supercapacitors based on onion-like carbon," Nature Nanotechnology, vol. 5, no. 9, pp. 651-654, 2010.

[11] S. Jiang, T. Shi, X. Zhan et al., "High-performance all-solid-state flexible supercapacitors based on two-step activated carbon cloth," Journal of Power Sources, vol. 272, pp. 16-23, 2014.

[12] Z. Niu, L. Zhang, L. Liu, B. Zhu, H. Dong, and X. Chen, "Allsolid-state flexible ultrathin micro-supercapacitors based on graphene," Advanced Materials, vol. 25, no. 29, pp. 4035-4042, 2013.

[13] J. Chmiola, G. Yushin, Y. Gogotsi, C. Portet, P. Simon, and P. L. Taberna, "Anomalous increase in carbon at pore sizes less than 1 nanometer," Science, vol. 313, no. 5794, pp. 1760-1763, 2006.

[14] M. Létiche, K. Brousse, A. Demortière et al., "Sputtered titanium carbide thick film for high areal energy on chip carbon-based micro-supercapacitors," Advanced Functional Materials, vol. 27, no. 20, Article ID 1606813, 2017.

[15] H. Long, T. Shi, S. Xi et al., "Growth of nano-wrinkles on photoresist-derived carbon microelectrode array," International Journal of Nanotechnology, vol. 11, no. 5-8, pp. 616-625, 2014.

[16] Y. Gao, T. Shi, H. Zheng, Z. Tang, and Q. Xia, "Formation of submicron buckling patterns in thin metal film on micro-scale substrate by pyrolysis of photoresist," Thin Solid Films, vol. 550, pp. 156-163, 2014.

[17] H. Li, J. Wang, Q. Chu, Z. Wang, F. Zhang, and S. Wang, "Theoretical and experimental specific capacitance of polyaniline in sulfuric acid," Journal of Power Sources, vol. 190, no. 2, pp. 578586, 2009.

[18] W. Chen, M. Beidaghi, V. Penmatsa et al., "Integration of carbon nanotubes to C-MEMS for on-chip supercapacitors," IEEE Transactions on Nanotechnology, vol. 9, no. 6, pp. 734-740, 2010.

[19] S. Xi, Y. Zhu, Y. Yang, S. Jiang, and Z. Tang, "Facile synthesis of free-standing $\mathrm{NiO} / \mathrm{MnO}_{2}$ core-shell nanoflakes on carbon cloth for flexible supercapacitors," Nanoscale Research Letters, vol. 12, no. 1, article 171, 2017.

[20] M. R. M. Jasni, M. Deraman, M. Suleman et al., "Effect of nano-scale characteristics of graphene on electrochemical performance of activated carbon supercapacitor electrodes," AIP Conference Proceedings. AIP Publishing, vol. 1710, no. 1, Article ID 030034, 2016.

[21] Y. Cui, L. Cheng, C. Wen, Y. Sang, P. Guo, and X. S. Zhao, "Capacitive behavior of chestnut shell-based porous carbon electrode in ionic liquid electrolytes," Colloids and Surfaces A: Physicochemical and Engineering Aspects, vol. 508, pp. 173-177, 2016. 


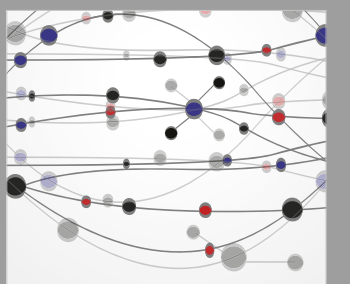

The Scientific World Journal
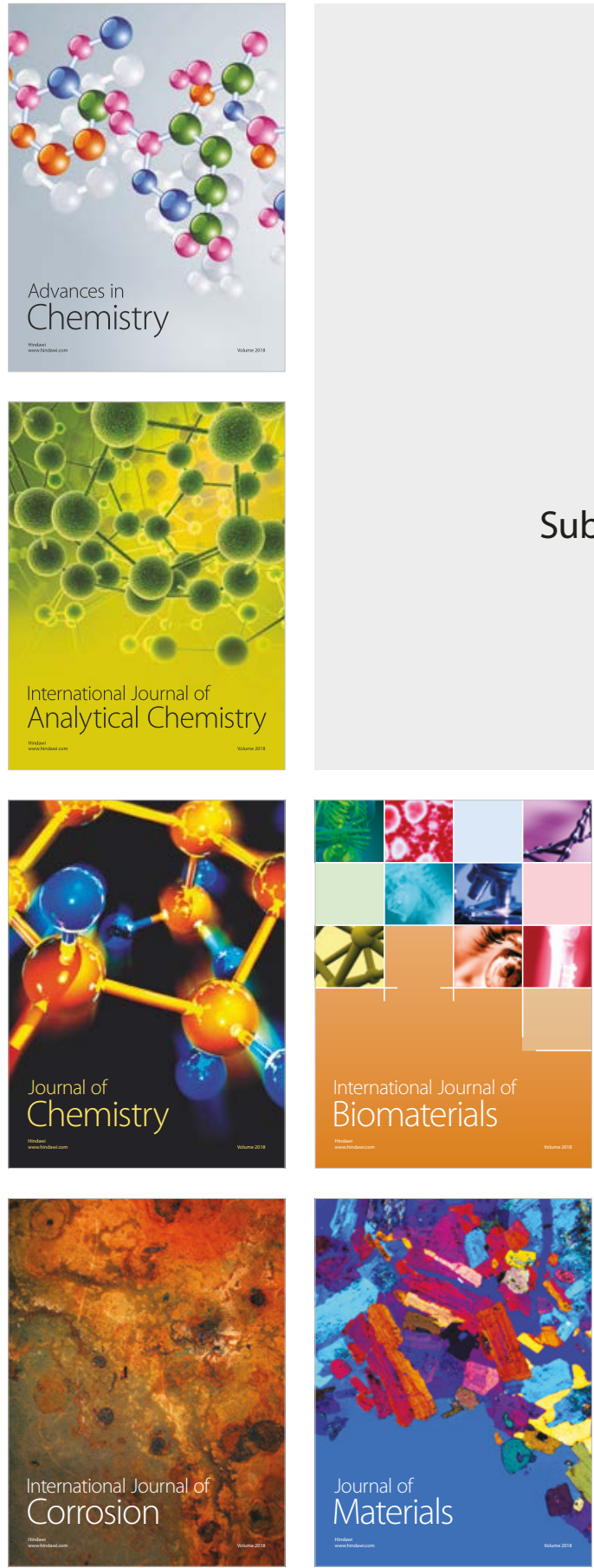

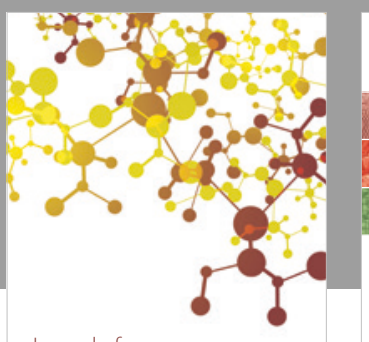

Journal of

Applied Chemistry
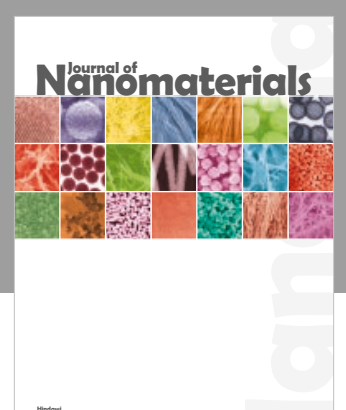

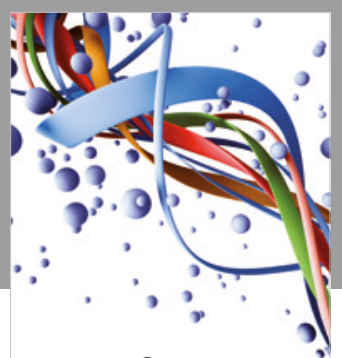

Scientifica

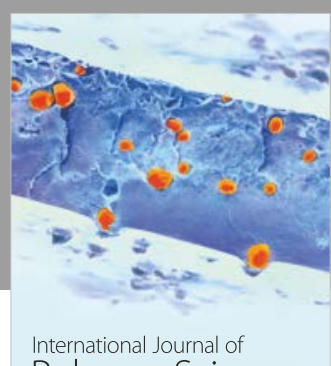

Polymer Science

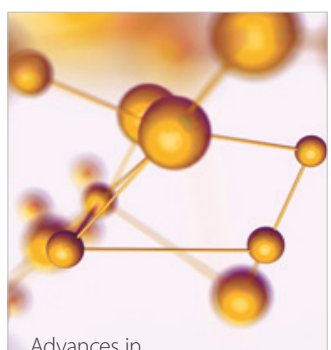

Physical Chemistry
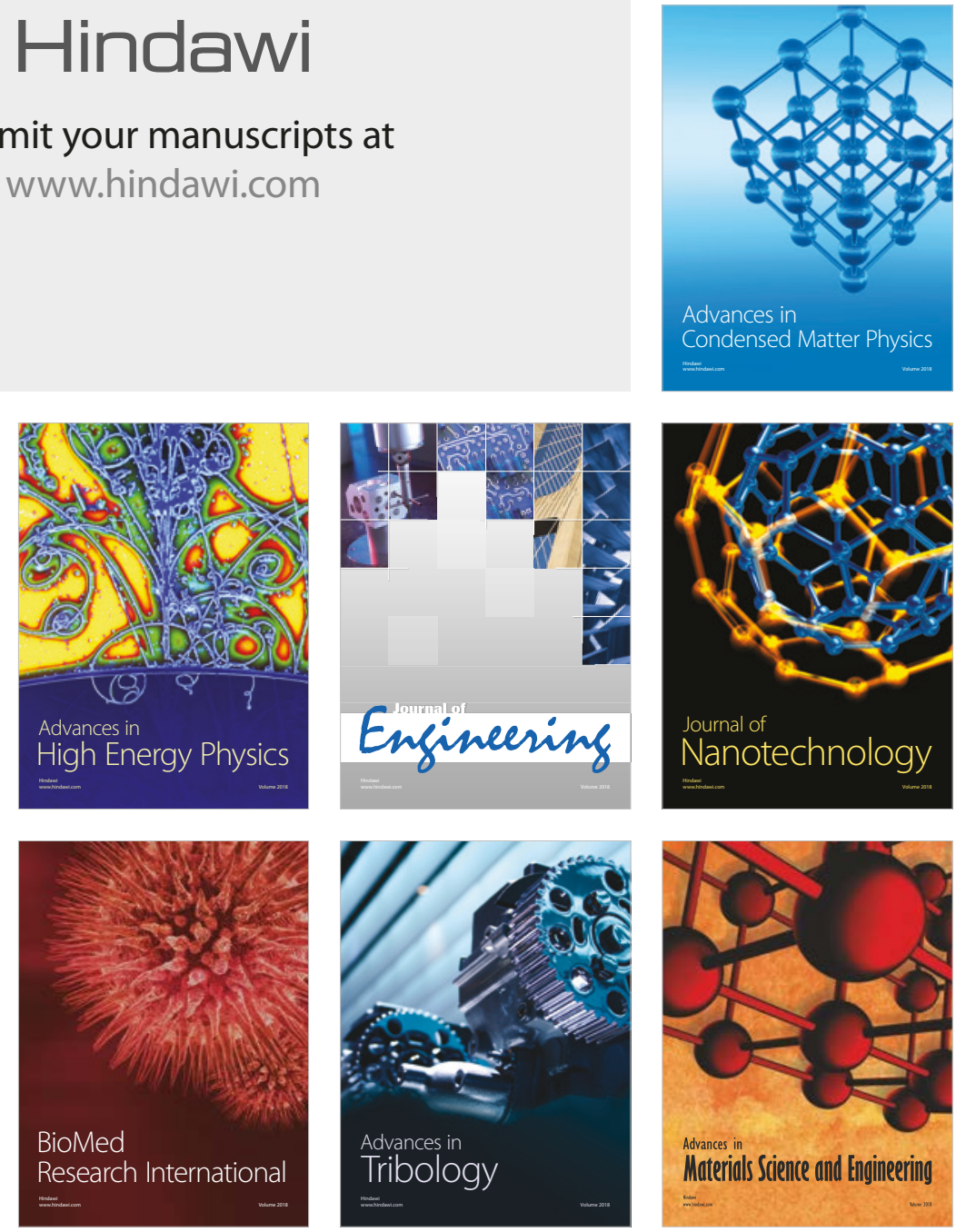